\title{
STUDIES ON THE APPLICATION OF VARIABLE AMPLITUDE LOADING TO CONCRETE THROUGH ENERGY EQUIVALENCE
}

\author{
K. KEERTHANA* AND J. M. CHANDRA KISHEN ${ }^{\ddagger}$ \\ Department of Civil Engineering \\ Indian Institute of Science, Bangalore, INDIA \\ *e-mail: keerthu@civil.iisc.ernet.in \\ ‡e-mail:chandrak@civil.iisc.ernet.in
}

Key words: Variable amplitude loading, Energy dissipation, Fatigue crack propagation

\begin{abstract}
Damage quantification of concrete subjected to variable amplitude load is not a simple task. In this study, a procedure is proposed to convert variable amplitude load acting on concrete structures to an equivalent constant amplitude load. The procedure described in this work is developed using an existing fatigue crack propagation model and an energy dissipation model which are both based on the energy approach. Further, the damage incurred by concrete is quantified using damage index, which is obtained by normalizing the cumulative energy dissipated with the total cumulative energy dissipated at the critical crack length of the concrete specimen.
\end{abstract}

\section{INTRODUCTION}

Engineering structures such as reinforced concrete buildings, bridges, pavements etc. are subjected to variable and non-proportional cyclic loads during their service. These loads cause failure under high cycle fatigue wherein the stress levels are relatively low leading to slow and gradual failure with large number of cycles and the deformations being elastic. Due to the non-uniformity in load and material properties, the computation of damage and the response of the structure becomes a very tedious process. Most of the fatigue tests conducted on the structural components to evaluate damage make use of constant amplitude loading, which reduces the complexity involved in the evaluation procedure but it fails to account for the variability involved in the service loads. If we could convert the in-service variable amplitude load to an equivalent constant amplitude load it would simplify the analysis procedure for the damage computation.

We need a suitable model to characterize the complex fatigue behavior of concrete. The conventional method to characterize the fatigue behavior of a material was through the use of S$\mathrm{N}$ curves. When dealing with variable amplitude loading, the counting algorithm such as rainflow [1] algorithm was used to count the number of cycles corresponding to a particular stress range present in the load history. Further, the Miner's [2] cumulative damage rule was applied to compute the damage $D$ which is given as:

$$
D=\sum \frac{n_{i}}{N_{f, i}}
$$

where $n_{i}$ and $N_{f, i}$ are, respectively, the applied number of cycles and the number of cycles to failure corresponding to the $i^{t h}$ constant amplitude of the stress range. The value of $D$ varies from 0 to 1 , where $D=1$ indicates that the material has undergone complete damage and $D=0$ indicates that the material has not undergone any damage. Miner's rule does not consider the effect of load sequence or load interaction. The experimental results on metals 
show $D>1$ for low to high loading sequence and $D<1$ for high to low loading sequence. In spite of its major drawbacks, this rule is still being used due to its simplicity. According to a review paper by Fatemi and Yang [3], many researchers have modified the linear damage rule in different ways to include the effect of load sequence and load interaction. Most of these damage rules are applicable only for metals. When concerned with concrete, the use of the linear damage rule or any other modified version of it would not give correct damage computation due to its heterogeneity. Oh [4], conducted experiments under variable amplitude loading on concrete and he proposed a nonlinear damage theory, which includes the effects of magnitude and sequence of applied fatigue loads. There are several approaches in which the fatigue phenomenon in concrete is studied, one of which makes use of the concepts of fracture mechanics. The study of crack propagation due to fatigue loading started with the well-known Paris'Law [5], wherein crack growth increment per load cycle is a function of applied stress intensity factor range. For the variable amplitude load, Barsom [6] proposed a statistical crack growth model, where he considered the root mean square value of stress intensity factor range in the Paris'Law equation. Other attempts have been made by researchers [7.-10],to model crack growth of concrete using Paris'Law. Linear elastic fracture mechanics based fatigue crack propagation law was proposed by Slowik et al. [11], which included parameters such as specimen size, load history and fracture toughness. This law was applicable to variable amplitude fatigue loading.

For quasi-brittle materials such as concrete, the physical mechanism of fatigue is characterized by debondings and micro-decohesions which give rise to microcracks. These microcracks coalesce to produce mesocracks and there is also a fracture process zone(FPZ) formed ahead of the crack tip which encapsulates various toughening mechanisms such as crack deflection, microcrack shielding, crack branching, crack tip blunting, grain bridging etc. [12]. In addition to the heterogeneity of concrete, when the variability of the load is included, the complexity becomes multifold. Consequently, the most appropriate approach to model the fatigue behavior of concrete is the energy based approach because it considers the energy balance and neatly avoids the analysis for singularity at the crack tip. The energy approach was adopted by many researchers for metals and brittle materials using the concepts of thermodynamics and damage mechanics. For concrete fatigue very few attempts have been made to quantify the damage under the thermodynamic framework. In the work done by Fathima and Chandra Kishen [13], a fatigue crack propagation model was proposed for concrete which was derived under the thermodynamic framework. This model was found to predict the fatigue crack propagation rate for different specimen sizes, strength and $\mathrm{R}$ ratios. In another work by Fathima and Chandra Kishen [14], a closed form expression for entropy generated during fatigue in terms of the energy dissipated was derived using the principles of dimensional analysis and self-similarity under the thermodynamic framework. The main drawback of these models was that, they were developed for constant amplitude load.

In the present work the existing fatigue crack propagation model [13] and the existing expression for dissipated energy [14] are considered for converting the variable amplitude loading to an equivalent constant amplitude load. A systematic procedure is developed for this conversion through energy equivalence. Finally, the damage incurred by concrete is quantified using normalized energy dissipated during the fatigue process.

\section{VARIABLE AMPLITUDE LOAD}

In this study, we consider a synthetic variable amplitude load (VAL) data, Figure 1 shows one block of VAL data. This block is repeatedly applied on to a concrete beam specimen as a flexural load at the mid span as shown in the Figure 2, until the crack length reaches its critical value. Synthetic VAL is applied on notched 
three-point bend concrete specimens of three sizes: small, medium and large.

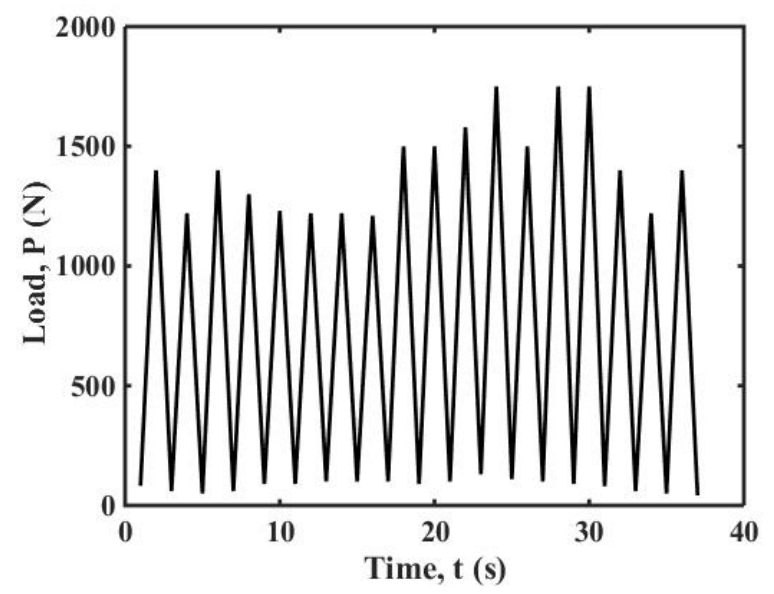

Figure 1: One block of synthetic VAL

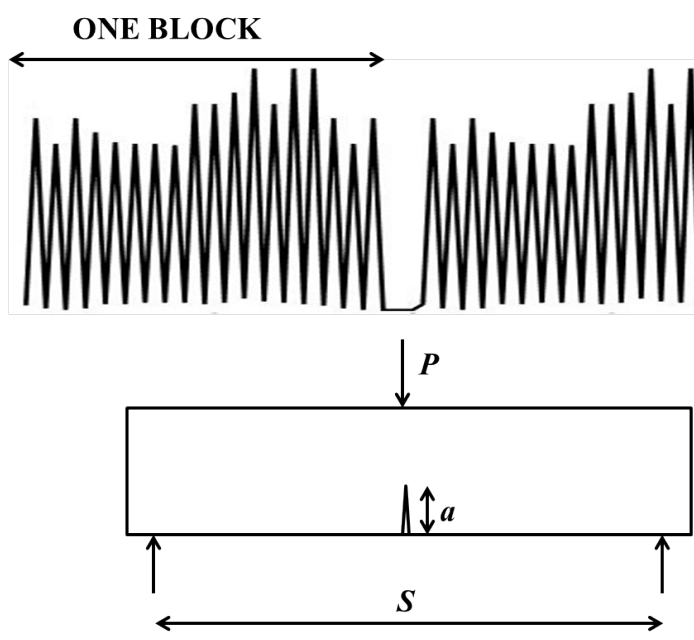

Figure 2: Synthetic VAL applied on three point bent concrete beam specimen

The geometry and material properties of the specimens are taken from Bazant and $\mathrm{Xu}[10]$ except for initial notch size which is taken as 25 percent of the depth in the present study. The specimen details are tabulated in Table 1 . For the small specimen, the VAL block shown in Figure 1 is applied, whereas for the medium and large specimens, the maximum amplitude is scaled up by 1.5 and 2.5 respectively. The frequency of the load is assumed to be constant for each cycle and its effect is not considered.

\section{FATIGUE CRACK PROPAGATION}

The fatigue crack propagation model proposed in [13] is used in this work. This model was derived using an energy approach within a thermodynamic framework and the principles of dimensional analysis and self-similarity. It is given as

$$
\frac{d a}{d N}=\gamma_{1} \Delta G_{I}^{\left(\gamma_{1}-1\right)} G_{F}^{\left(-\gamma_{1}-\gamma_{2}\right)} f_{t}^{\left(1-\gamma_{2}\right)} a^{\gamma_{2}}
$$

where $\Delta G_{I}=$ energy release rate range for mode I loading; $G_{F}=$ fracture energy; $f_{t}=$ tensile strength of concrete; $a=$ crack length and $N=$ number of load cycles. The unknown constants $\gamma_{1}$ and $\gamma_{2}$ were determined in [13] through a calibration process using the experimental results of [10] and multivariate regression was performed and the following expressions were obtained for $\gamma_{1}$ and $\gamma_{2}$.

$$
\begin{aligned}
& \gamma_{1}=-2.213 R+\left(1.45 f_{t}^{2}-12.58 f_{t}+30.55\right) \\
& \gamma_{2}=-5.048 R-0.7
\end{aligned}
$$

where $R$ is the load ratio. The energy release rate range, $\Delta G_{I}$ is computed using the following expression.

$$
\Delta G_{I}=\frac{\Delta P^{2}}{2 B} \frac{d C}{d a}
$$

where $\Delta P=P_{\max }-P_{\min }$ is the load range and $P_{\text {max }}$ and $P_{\text {min }}$ are the maximum and minimum values of the fatigue load cycles; $B=$ width of the beam; and $d C / d a=$ rate of change of compliance with respect to the crack length. In the present study this crack propagation model is used to compute the increase in crack length when the specimen was subjected to a known number of load cycles.

\section{ENERGY DISSIPATION}

The energy dissipated during the fatigue process in concrete must include the energy absorbed to form two new surfaces as the crack advances, and the energy dissipated in the FPZ where various toughening mechanisms take 
Table 1: Geometry and material properties of beam specimens

\begin{tabular}{cccccccc}
\hline \multirow{2}{*}{ Specimen } & $\begin{array}{c}\text { Depth } \\
\mathrm{D}\end{array}$ & $\begin{array}{c}\text { Span } \\
\mathrm{S}\end{array}$ & $\begin{array}{c}\text { Thickness } \\
\mathrm{B}\end{array}$ & $\begin{array}{c}\text { Notch size } \\
a_{0}\end{array}$ & $G_{F}$ & $f_{t}$ & Peak Load \\
& $m m$ & $m m$ & $m m$ & $m m$ & $N / m m$ & $N / m^{2}$ & $N$ \\
\hline Small & 38.1 & 95 & 38.1 & 9.5 & 0.049 & 2.86 & 1815 \\
Medium & 76.2 & 191 & 38.1 & 19.0 & 0.083 & 2.86 & 2986 \\
Large & 152.4 & 381 & 38.1 & 38.1 & 0.134 & 2.86 & 5184 \\
\hline
\end{tabular}

place. Considering these factors, the expression for energy dissipation per unit volume of a specimen was derived in a previous work [14], using a thermodynamic framework in conjunction with the principles of dimensional analysis and self-similarity. Energy dissipated per unit volume, $E_{d}$, is a function of the energy release rate range $\Delta G_{I}$, load ratio $R$, compliance of the specimen $C$ and the length of FZP $l_{c h}$ ahead of the crack tip in the direction of crack propagation. It is given as

$$
E_{d}=\Delta G_{I}^{0.9} C^{-0.1} l_{c h}^{-1}(1-R)^{2.6}
$$

The compliance for a given crack length required in Eqs. 5 and 6 is computed using a procedure adopted from Bazant and Planas [15]. The energy release rate $G$ is related to the stress intensity factor $K_{I}$ according to Irwins equation, as

$$
G=\frac{K_{I}^{2}}{E}
$$

For a three point bend beam, Eq. 7 can be written as

$$
\Delta G_{I}=\frac{\Delta P^{2}}{2 B} \frac{d C}{d a}=\frac{9 \Delta P^{2} S^{2} \pi a f(\alpha)^{2}}{4 E B^{2} D^{4}}
$$

where $C=$ compliance of the member; $\alpha=$ relative crack depth defined by $a / D$, where $a=$ crack length; $D=$ depth of the specimen; $f(\alpha)=$ geometry factor; and $S=$ span of the member. From the above equation the compliance is computed as

$$
C=\frac{9 S^{2} \pi}{2 B D^{2} E} \int_{0}^{a / D} \alpha f^{2}(\alpha) d \alpha+C_{0}
$$

where $C_{0}=$ compliance of the uncracked body. The length of the FPZ $\left(l_{c h}\right)$ ahead of the crack tip, also called the material characteristic length, is given as

$$
l_{c h}=\frac{E G_{F}}{f_{t}^{2}}
$$

where $E=$ modulus of elasticity of concrete; $G_{F}=$ fracture energy; and $f_{t}=$ tensile strength of concrete. The load ratio $R=P_{\min } / P_{\max }$. Using Eqs. 9, 10, load ratio $\mathrm{R}$ and the increased value of crack length computed from Eq. 2 in Eq. 6, the energy dissipated per unit volume of a specimen can be obtained.

\section{CONSTANT AMPLITUDE LOAD}

The objective of the present work is to convert the variable amplitude loading to an equivalent constant amplitude load (CAL) history for application to concrete structures. To start with, we assume the maximum amplitude $\left(P_{\max }\right)$ and the minimum amplitude $\left(P_{\text {min }}\right)$ of the equivalent CAL as the algebraic mean of all the maximum and minimum values of VAL, respectively. The number of cycles of the equivalent CAL being an unknown is determined through an energy equivalence approach as discussed in the following section.

\section{CONVERSION OF VAL TO CAL THROUGH ENERGY EQUIVALENCE}

In this section, a procedure developed to convert the synthetic VAL used in this study to an equivalent CAL through energy equivalence is described in the following steps:

1. For each cycle of VAL, the increase in crack length is computed using Eq. 2.

2. The increased value of crack length is substituted in Eq. 6 through the expres- 
Table 2: Results of variable amplitude load and constant amplitude load after the application of conversion procedure

\begin{tabular}{ccccccccc}
\hline & \multicolumn{3}{c}{ Variable amplitude load } & \multicolumn{5}{c}{ Constant amplitude load } \\
Specimen & Blocks & $E_{d}^{V A L}$ & $a_{c}$ & $P_{\max }$ & $P_{\min }$ & Cycles & $E_{d}^{C A L}$ & $a_{c}$ \\
& $N_{b}$ & $N / m m^{2}$ & $m m$ & $N$ & $N$ & $N_{e q}$ & $N / m m^{2}$ & $m m$ \\
\hline Small & 26485 & 34.92 & 23.9 & 1676 & 80 & 508013 & 34.96 & 23.8 \\
Medium & 184444 & 164.52 & 48.1 & 2748 & 80 & 1939060 & 164.49 & 48.2 \\
Large & 244309 & 206.70 & 79.0 & 4590 & 80 & 2690853 & 206.77 & 79.1 \\
\hline
\end{tabular}

sion of $\Delta G_{I}$ given in the Eq. 8 to compute the energy dissipated per unit volume of the specimen.

3. The steps (1) and (2) are repeated for each cycle of VAL until the critical crack length $\left(a_{c}\right)$ of the specimen is reached and the cumulative energy dissipated $\left(E_{d}^{V A L}\right)$ up to critical crack length is calculated. The critical crack length is the length at which the crack becomes unstable at certain applied load.

4. For the CAL, assume initial values of $P_{\max }$ and $P_{\min }$ as described in section 5.

5. Repeat the steps (1) and (2) for CAL to compute the increase in crack length and the energy dissipated per unit volume.

6. The step (5) is repeated until the critical crack length is reached. The corresponding cumulative energy dissipated per unit volume $\left(E_{d}^{C A L}\right)$ and the equivalent number of cycles $\left(N_{e q}\right)$ of CAL required are obtained.

7. Check for energy equivalence: The energy dissipated per unit volume up to critical crack length should be equal for both VAL and CAL, i.e. $\left(E_{d}^{V A L}\right)=\left(E_{d}^{C A L}\right)$.

8. If $\left(E_{d}^{V A L}\right) \neq\left(E_{d}^{C A L}\right)$, go to step (4), change the initially assumed value of $P_{\max }$ and $P_{\min }$ of CAL and repeat the steps (5) to (7) till the energy equivalence is achieved.
9. Once the energy equivalence is achieved, VAL is converted to CAL with $N_{e q}$ number of cycles.

In the present work, the above procedure was applied for three specimens: small, medium and large. VAL applied on them was converted to an equivalent CAL. The results are tabulated in Table 2. The variation of compliance with respect to crack length is shown in Figure 3. It is seen from this figure that the increase in compliance with crack length matches closely for VAL and CAL for all three specimens, thereby validating the energy equivalence concept.

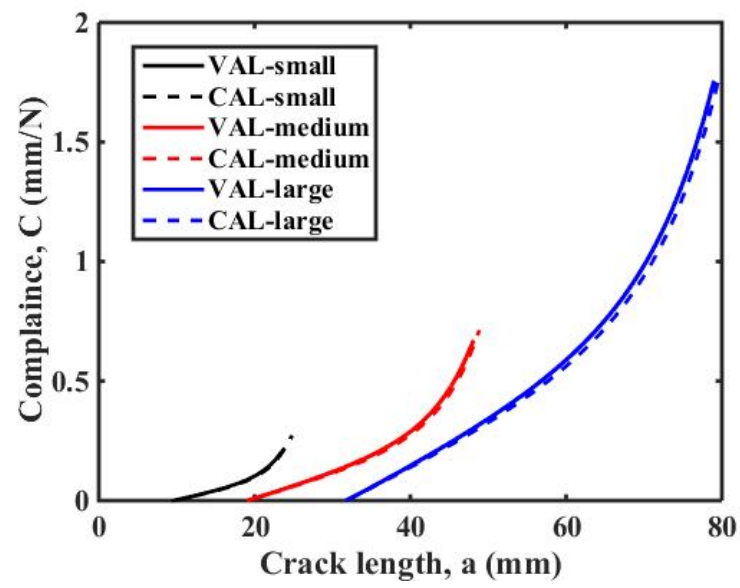

Figure 3: Variation of compliance with respect to crack length for beam specimens

\section{DAMAGE INDEX}

In this section damage index $(D)$ for a concrete specimen is defined with respect to the normalized cumulative energy dissipated per unit volume. It is given as

$$
D=\sum \frac{E_{d, i}}{E_{d f}}
$$


where $E_{d, i}$ is the cumulative energy dissipated with respect to the $i^{t h}$ cycle of CAL and $E_{d f}$ is the total energy dissipated at final fatigue failure. It is considered as a material property independent of loading and specimen size, as given in [14]. The value of the $D$ varies between 0 and 1 , where $D=0$ corresponds to no damage or no increase in the crack length or no energy dissipation. $D=1$ corresponds to complete damage or crack length reached its critical value or cumulative energy dissipated $\left(E_{d}\right)$ is equal to the total energy dissipated up to critical crack length $\left(E_{d f}\right)$. Unlike cumulative linear damage rule, the Eq. 11 is not based on the number of cycles to failure at a paritucular stress range. The damage index computed here is based on the energy dissipated as the crack propagates, which is directly derived from the first principles using thermodynamic approach and dimensional analysis. Since the damage index computed is based on energy, the expression encapsulates the effect of sequence and the magnitude of VAL implicitly. The variation of damage index as a function of relative crack depth and as a function of number of cycles of equivalent CAL $\left(N_{e q}\right)$ for three geometrically similar specimens is shown in Figures 4 and 5 respectively. As expected, the damage index increases with the increase in crack length and number of cycles with the rate of increase being relatively higher while approaching failure.

\section{CONCLUSIONS}

The damage quantification of concrete subjected to variable amplitude load is a complex process due to the variability in load and the heterogeneity of the material. In this study, an attempt has been made to convert the variable amplitude load to an equivalent constant using an energy equivalence concept. A systematic procedure is developed for this conversion using existing fatigue crack propagation and energy dissipation models both of which were derived using a thermodynamic framework. Once equivalent constant amplitude is obtained the analysis becomes simpler and the damage in concrete could be easily quantified. Further work needs to be done to validate the developed procedure with experimental results.

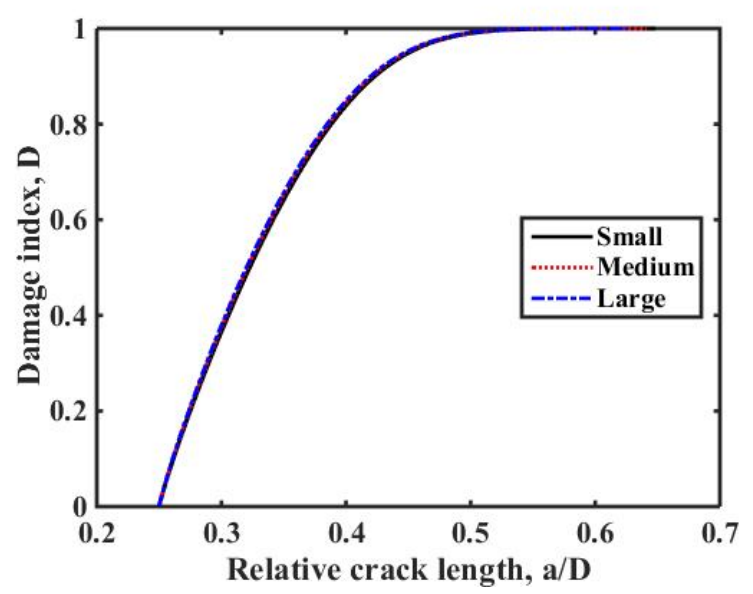

Figure 4: Variation of Damage index with respect to relative crack depth

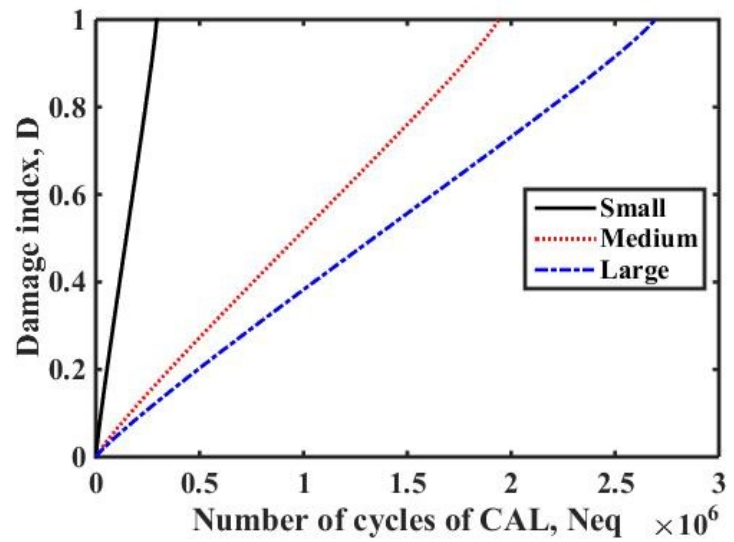

Figure 5: Variation of Damage index with respect to equivalent number of CAL cycles

\section{REFERENCES}

[1] ASTM-E1049-85, 1990. Standard Practices for Cycle Counting in Fatigue Analysis. West Conshohocken, PA: American society for testing and materials.

[2] Miner, M.A., 1945. Cumulative damage in fatigue. Journal of applied mechanics 12(3), pp.159-164.

[3] Fatemi, A. and Yang, L., 1998. Cumulative fatigue damage and life prediction 
theories: a survey of the state of the art for homogeneous materials. International journal of fatigue. 20(1), pp. 9-34.

[4] Oh, B.H., 1991. Cumulative damage theory of concrete under variable-amplitude fatigue loadings. ACI Materials Journal, 88(1), pp.41-48.

[5] Paris, P.C. and Erdogan, F., 1963. A critical analysis of crack propagation laws. Journal of basic engineering, 85(4), pp.528-533.

[6] Barsom, J.M., 1976. Fatigue crack growth under variable-amplitude loading in various bridge steels. In Fatigue Crack Growth Under Spectrum Loads. ASTM International.

[7] Swartz, S.E., Jones, G.L. and Hu, K.K., 1978. Compliance monitoring of crack growth in concrete. Journal of the Engineering Mechanics Division, 104(4), pp.789-800.

[8] Perdikaris, P.C. and Calomino, A.M., 1989. Kinetics of crack growth in plain concrete.In Fracture of concrete and rock (pp. 64-69). Springer New York.

[9] Baluch, M.H., Qureshy, A.B. and Azad, A.K., 1989. Fatigue crack propagation in plain concrete.International conference on Fracture of concrete and rock, Springer-verlag, New York. 80-85.

[10] Bazant, Z.P. and Xu, K., 1991. Size effect in fatigue fracture of concrete.ACI Materials Journal, 88(4), pp.390-399.

[11] Slowik, V., Plizzari, G.A. and Saouma, V.E., 1996. Fracture of concrete under variable amplitude fatigue loading.ACI Materials Journal, 93, pp.272-283.

[12] Shah, S.P., Swartz, S.E. and Ouyang, C., 1995. Fracture mechanics of concrete: applications of fracture mechanics to concrete, rock and other quasi-brittle materials. John Wiley \& Sons.

[13] Pervaiz Fathima, K.P. and Chandra Kishen, J.M., 2013 A thermodynamic framework for fatigue crack growth in concrete. International Journal of Fatigue,54, pp.17-24.

[14] Pervaiz Fathima, K.P. and Chandra Kishen, J.M., 2015. Prediction of Fatigue Life in Plain concrete Using Entropy Production. Journal of Engineering Mechanics, 141(7), p.04015007

[15] Bazant, Z.P. and Planas, J., 1997. Fracture and size effect in concrete and other quasi brittle materials (Vol. 16). CRC press. 